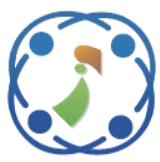

\title{
Design, Development and Analysis of Variable Bandwidth Filter Bank for Enhancing the Performance of Hearing Aid System
}

\author{
Ujjwala S. Rawandale ${ }^{1 *}$ \\ Mahesh T Kolte ${ }^{2}$ \\ ${ }^{I}$ Electronics and Communication Engineering Department, MIT World Peace University, \\ Pune, Maharashtra - 411038, India and Research Scholar at SCOE, Pune \\ ${ }^{2}$ Electronics and Telecommunication Engineering Department, Pimpri Chinchwad College of Engineering, \\ Pune, Maharashtra - 411044, India \\ *Corresponding author's Email: ujjwala.rawandale@mitwpu.edu.in
}

\begin{abstract}
The hearing aid system is used for hearing impair patient communication, but the hearing aid's main issues are improper communication, power dissipation, delay, matching error, and low complexity. Many existing filter bank techniques are developed to overcome these issues but still have the problem of delay, unwanted noise, etc. This paper has designed a Tuned Feed forward Variable Bandwidth Filter (TFVBF) to enhance the Hearing Aid System (HAS) by reducing unwanted noise, matching error, and complexity. Moreover, the developed technique is implemented in MATLAB and it achieve low rate in power consumption and delay. Thus, the gained performance metrics has enhanced the HAS's performance by reducing, unwanted noise, matching error, and complexity. Finally, the attained performance metrics of the developed technique using 50 bands are compared with existing replicas in terms of matching error, delay, power consumption, and complexity for checking the effectiveness of developed replica.
\end{abstract}

Keywords: Hearing aid, Multipliers, Sub filters, Hearing impair patient, Audio signal, Communication, Filter, Noise.

\section{Introduction}

Signal processing is the subfield of electrical engineering, and it is mainly focused on modifying, analyzing, and synthesizing signals in terms of images, sounds, and scientific calculations [1, 2]. Moreover, signal processing methods are used for enhancing transmission, quality, storage efficiency, and highlight or detect the interest components of the calculated signal [3]. Additionally, signal processing contains frequency domain, time domain, and complex frequency domain. The signal processing categories are analog, discrete-time, nonlinear, and digital, continuous $[4,5]$. Some application of signal processing is video processing, array processing, image processing, feature extraction, wireless communication, audio signal processing and so on [6]. The architecture of signal processing has illustrated in Fig. 1 [7]. One of the most wanted signal processing applications is the
HAS it will use by the hearing-damaged user for communication [8].

Moreover, HAS is the most common research through the manufacture of Hearing Aid (HA) at a proprietary system [9]. Providing open tools in HA is the biggest barrier for signal processing [10]. In addition, the platform of software is developed to enhance the HAS [11]. It will provide a complete set of HA signal processing and enable researchers to perform offline processing with less delay and significant processing power [12]. The advantage of developed HAS is that it will run a large range of hardware and perform better in battery power, efficient power, delay, and headless $[13,14]$. The main aim of the HA is for improving speech by understanding certain individuals of hearing impair patients [15]. As well, digital HA is more complex because it will collect the sound and converted it into digital signals, and the digital signals are transferred to the computer chip of the hearing aid 

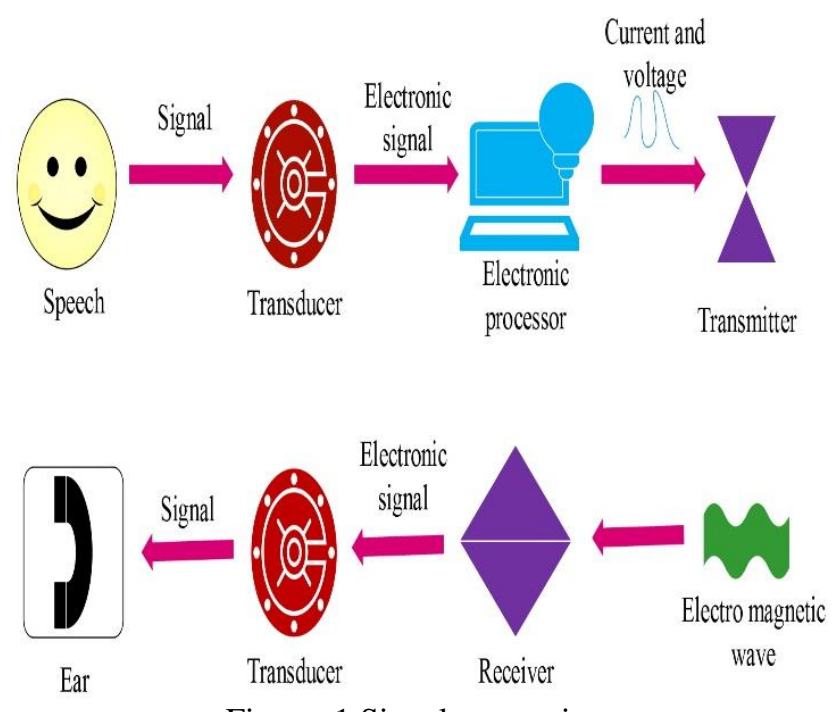

Figure. 1 Signal processing

[16]. Then it will convert into acoustic sound, but the issues of HAS are noise, improper communication, complexity, delay, power dissipation, and so on [17].

As a result, many techniques are developed to enhance the HAS [18] and reduce the problem of power dissipation, noise reduction, delay, matching error, and better performance in the communication device of HAS [19]. Thus the developed existing techniques are reconfigurable HA [21], digital HAS [22], less complexity technique [23], and analog correlator [25] but still having the issues of HAS. So in this paper proposed a new framework for optimizing the performance of HAS [20].

The structure of this paper is summarized below: The related work of this research is summarized in Section 1. The system model and the problem statement are explained in Section 2. The proposed method is elaborated in Section 3. Consequently, the result and discussion, along with the comparative analysis, are described in Section 4. Finally, the paper is concluded in Section 5.

\section{Related works}

Tomson Devis and Manju Manuel [21] have proposed a new reconfigurable HA to reduce the complexity by auto adapting ability. It will be suitable for various kinds of hearing disabilities assortment as of mild to serve up intensities. Also introduced audio spectrum with three areas and the three areas contain four schemes. Moreover, automatic selection of optimum techniques is developed on the hearing threshold. Thus, the developed technique is implemented in the hardware platform it will avoid manual interventions and reduce the time for matching audiograms but contain large noise during A/D convention.
The filter bank has been placed a major role in designing digital HAS, and the suitable structure of the HAS is developed. Mahesh and Shahana [22] have developed a finite impulse response filterbased smooth transition characteristics technique and minimum phase filter technique to reduce the filter coefficient. It will exhibit less pass band delay by the same magnitude response. Also, the developed technique reduces the hardware complexity, but it has required more time to convert audiograms.

Devis and Manuel [23] have introduced less complexity to decompose the sub-band of the audio signal to HA. The main aim of the developed technique is to design an audibility restoration application. In this technique, three capable filter banks are introduced. The fractional interpolation method is used to generate more quantities of sub bands through narrow bandwidth. Thus the filter bank is used for hearing injury of patients. Hence it has been a loss of high power dissipation.

The low complexity of interpolated restricted impulse response depends on 18 bands for HA is introduced Deepu et al. [24]. Thus, the developed technique attains the maximum available margin at the filter specification and is utilized in all lower and upper band bandwidths. The main aim of the developed method is to reduce the order of the filter and reduce computation time. The filter specification has been attained more matching errors and high preprocessing time because of the large bandwidth.

Hyusim Park et al. [25] have proposed an analog correlator of the front end by the control circuit of HAS. Thus the developed analog front end contains a compressed analog correlator multiplier, variable gain amplifier, and integrator. Moreover, analog correlator gain low referred input noise, and the implemented gain control is used to gain amplifier. The developed technique attains a wide dynamic range and low noise while comparing other techniques; also, the performance of HAS is enhanced but has high complexity. below:

The key steps of the approach are discussed

- Initially, design and developed TFVBF to the HAS.

- Hereafter, original speech audio signal (analog signal) is trained to the system.

- Moreover, the developed technique is implemented in MATLAB environment.

- Consequently, the filter parameter was developed to enhance hearing aid performance in terms of delay, matching 
error, complexity, power dissipation, and so on.

- Finally, gained performance metrics of the developed replica is compared with another existing method in respect of complexity, delay, matching error and power consumption.

\section{System model and problem statement}

Generally, the microphone converts the sound into an analog electrical signal, and the signal is sent to the anti-aliasing filter. Thus the anti-aliasing filter removes the unclear frequencies, and the sampled signal permits electrical signal into Analog to Digital (A/D) converter. Thus, the converted A/D signals are transferred to the digital signal processing containing a microprocessor to operate the digital signal. The next Digital to Analog (D/A) signal converts the digital signal into an analog signal. Then the signal is sent to the anti-imaging filter to smooth the signal and obtains natural sound. The receiver converts the signal into a hearing aid, but the generated sound contains a high error, delay, and power dissipation. Moreover, the system model and problem definition have illustrated in Fig. 2.

The main problem of HAS is high frequency, sound louder, minimize matching error, low complexity, high noise rate, and grabbed speech. For overcoming these issues, several techniques are developed but still having the problems of noise, improper communication, delay, complexity, and power dissipation. So a novel TFVBF is developed for reducing unwanted noise, delay, the error also attain low complexity. The main aim of the HA is to improve speech by understanding certain individuals of hearing impair patients.

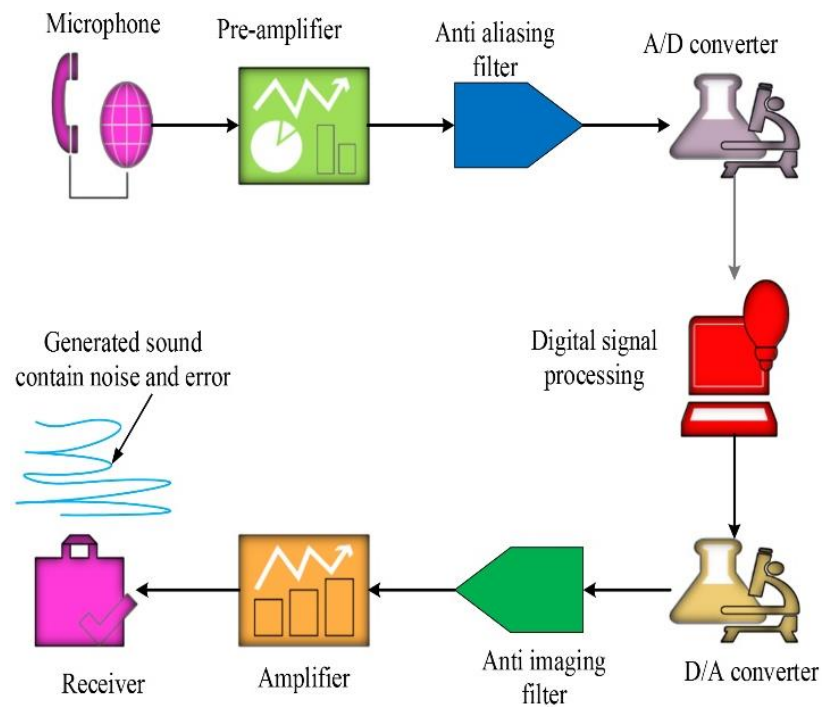

Figure. 2 System model and problem statement

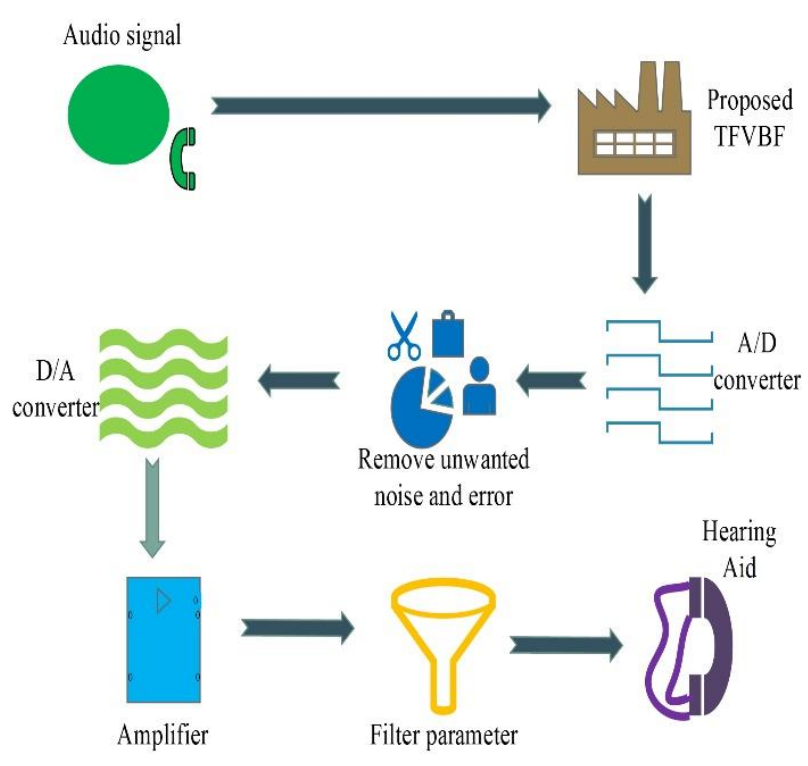

Figure. 3 Proposed TFVBF methodology

\section{Proposed methodology}

Nowadays, digital HA is mainly useful for hearing damage, but the main issues of the HAS are error, unwanted noise, delay, and high complexity. Many existing methods are developed to enhance the HAS but have the issue of matching error, noise, delay, power dissipation, and high complexity. So, this paper proposed a new design of a TFVBF to enhance HAS's performance and remove unwanted noise, delay, power dissipation, and errors present in the audio signal. Finally, the performance metrics of the developed replica is compared work other existing technique for checking the efficiency of the proposed technique. The process of the proposed TFVBF technique has illustrated in Fig. 3.

Initially, the audio signals are tuned to the TFVBT, and the tuned analog signals are converted into digital signals. Next, using the developed TFVBF removes all unwanted noise, delay, power dissipation, and matching errors presented in the signal. Moreover, cleaned digital signals are converted into analog signals using the amplifier and filter parameter. Finally, the filtered signal is sent to the hearing aid system. Developed replica enhances HAS's performance and reduces delay, error, power dissipation, and unwanted noise.

\subsection{Design of Tuned Feed forward Variable Bandwidth Filter (TFVBF)}

The developed TFVBF technique is designed to optimize HAS, and the proposed filter bank is proposed to reduce noise, delay, matching error, and complexity. Thus the developed technique matches the wide range of audiograms, and the advantage of 


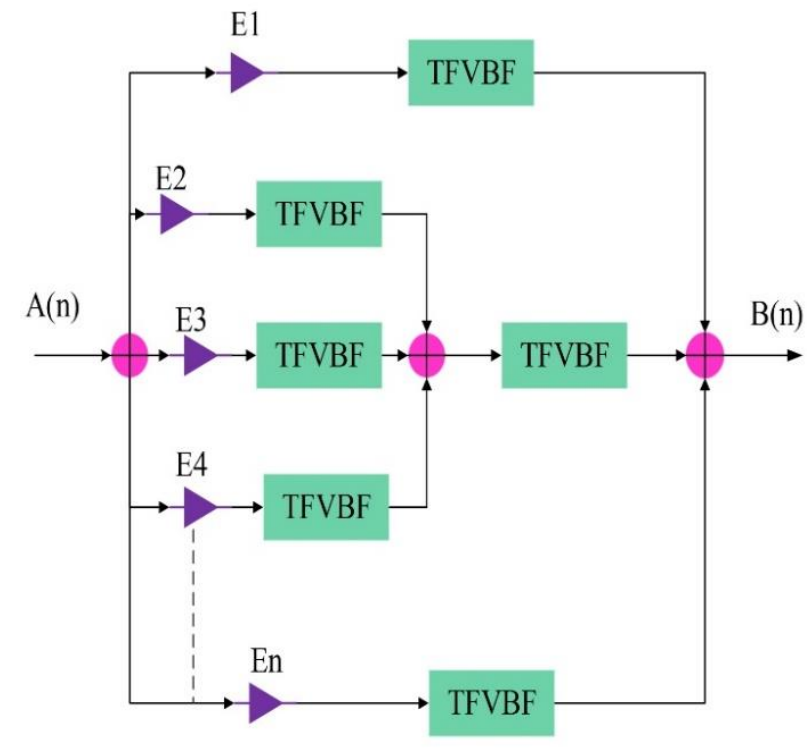

Figure. 4 Design of developed TFVBF

TFVBF is mass production. In this technique, a large set of bandwidths is used to fit the audiograms selected from the hearing loss pattern. Moreover, bands produced the developed TFVBF to attain essential frequency through frequency shifts. Furthermore, the selection of frequency and gains are used for minimizing the delay, power dissipation, and matching error. The resultant matching curve follows the shape of every audiogram, and the matching error is the difference between audiograms and the matching curve. The design of the developed TFVBF has illustrated in fig. 4. For improving the performance of hearing aid tuned feed forward filter is introduced. The process of TFVBF will reduce and control the errors, delay, power dissipation, unwanted noise and complexity.

The determination of designed tuned filter parameter is denoted as $k_{f}=a-b$, where, $k_{f}$ is denoted as the designed feed filter and a and $b$ are considered as an audio signal.

Moreover, the reduced rate error and delay in the signal processing is calculated using Eq. (1),

$$
E_{a}=\frac{\left(P_{a-1}-Q_{a} P_{a} C_{a, a-1}\right)}{1+Y_{a} Q_{a} P_{a}} S_{a-1}
$$

Let, $E_{a}$ is the transfer function of error presented in the audio signal and $P_{a}$ is denotes as phase response. Also $Q_{a}$ is considered as closer priority of the filter. Moreover, $Y_{a}$ is denoted as the order of phase response and $S_{a}$ is expressed as a sub-band filter. Thus the removal of error has been obtained using Eq. (2),

$$
C_{a, a-1}=\frac{P_{a-1}}{Q_{a} P_{a i}}
$$

Let, $C_{a, a-1}$ is represented the removal of error. By using Eq. (2) easily removing the unwanted noise, delay, and error present in that signal and the substitution obtained value is $E_{a}=0$. While the transfer function of the error is $k_{f}>1$ means it will control the errors in a signal using Eqs. (3) and (4),

$$
\begin{gathered}
E_{a}=\frac{\left(P_{a-1}-Q_{a} P_{a} C_{a, a-1}\right) S_{a-1}-\sum_{a=b-k_{f}}^{a-2} Q_{b} P_{b} C_{a, b} S_{b}}{1+Y_{a} Q_{a} P_{a}} \\
U_{a}=\frac{\left(C_{a, a-1}+Y_{a} P_{a-1}\right) S_{a-1}-\sum_{a=b-k_{f}}^{a-2} C_{a, b} S_{b}}{1+Y_{a} Q_{a} P_{a}}
\end{gathered}
$$

Where, $U_{a}$ is denoted as the control ratio of errors. The substitution of Eqs. (3) and (4) obtain Eq. (5). In combination, they remove the unwanted noise, errors present in the tuned audio signal.

$$
\frac{E_{a}}{U_{a}-k_{f}}=-\sum_{b=a-k_{f}}^{a-2} \frac{X_{a, b}}{H_{a, b}} \prod_{a=i-k_{f}+1}^{a-1} B_{i}^{i+1}
$$

Let, $B_{i}^{i+1}$ represented as fractional delay and $X_{a, b}$ is the polynomial function of error rate. Moreover, $H_{a, b}$ is expressed as a generalized function of sub filter. Consequently, the frequency response of TFVBF is determined using Eq. (6),

$$
C_{a, b}=\frac{1+Y_{a} Q_{a} P_{a}}{Q_{a} P_{a}} \times \frac{X_{a, b}}{H_{a, b}}, b \neq a-1
$$

$C_{a, b}$ is denoted as the frequency response of TFVBT; it will filter the analog signal into a digital signal. Based on the input audio signal, it will change the velocity of a digital signal. And the frequency response of input and output signal is given as Eq. (7),

$$
C f_{a, b}=\frac{a(n)}{b(n)}
$$

Where, $C f_{a, b}$ is denoted as the frequency response of input and output signal measurement and $a(n)$ is called as input audio signal also $b(n)$ is represented as output audio signal. Moreover, the sub-filter bandwidth of TFVBF is calculated using Eq. (8),

$$
V_{a-1}^{a}=\frac{S_{a}}{S_{a-1}}=\frac{1}{Q_{a} P_{a}}\left(P_{a-1}+\sum_{b=a-k_{d}}^{a-2} \frac{X_{a, b}}{H_{a, b}} \frac{1}{\prod_{i=k}^{a-2} B_{i}^{i+1}}\right)
$$

Where, $V_{a-1}^{a}$ is denoted as a sub-filter bandwidth of TFVBF and designed TFVBF is carrying the same or various order based on the requirements. Thus the various sub-filters can minimize the complexity and high frequency. Moreover, the 
reduction of complexity is gained through multiplier replacing. Thus the replacements have happened with the help of shifter and adders. The replacement of multiplier is attained using Eq. (9).

$$
C_{a, a-k_{d}}=\frac{1+Y_{a} Q_{a} P_{a}}{Q_{a} P_{a}} \times \frac{n}{\prod_{i=1}^{3+k_{d}}\left(B_{i}^{a, a-k}\right) n+1}
$$

Let, $C_{a, a-k_{d}}$ is represented as the design of sub filters and $B_{i}^{a, a-k_{d}}$ is denoted as the replacement of multiplier. Additionally, the workflow of TFVBF is detailed in algorithm 1.

Finally, the estimation of filter parameter is determined using Eq. (10).

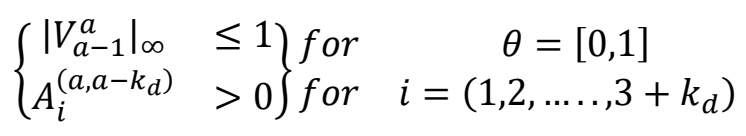

The estimated parameter is developed in the bandwidth filter bank, such as error, complexity, delay, power dissipation, unwanted noise, and matching error. Finally, the output is successfully updated in HAS with no error and unwanted noise. Moreover, developed TFVBF improve the performance of hearing aid and reduce the complexity

Also, it will remove unwanted noise, errors present in the input audio signal. This proposed technique is mostly useful for hearing loss patients. Thus the workflow of the developed TFVBF has detailed in Fig. 5.

\begin{tabular}{ll}
\hline \multicolumn{2}{c}{ Algorithm 1 Proposed TFVBF } \\
\hline Start
\end{tabular}

\section{Design and developed TFVBF}

// design filter bank structure

Initialize filter parameters $a$ and $b$ to proposed TFVBF

$k_{f}=a-b$

I/ $a$ and $b$ are the input audio signals

$k_{f}$ - designed feed filter

Converted into a digital signal

Remove and control the error rate

// it will filter unwanted noise, error, and matching error
$\frac{E_{a}}{U_{a}-k_{f}}=-\sum_{b=a-k_{f}}^{a-2} \frac{X_{a, b}}{H_{a, b}} \prod_{a=i-k_{f}+1}^{a-1} B_{i}^{i+1}$

$I / X_{a, b}$ - polynomial function of error rate

$H_{a, b}$-generalized function of sub-filter

$\boldsymbol{I} \boldsymbol{f}\left(k_{f}>1\right)$

I

Control and reduce errors

\}

Else $\left(E_{a}=0\right)$

I

No error

\}

Update to transfer sub-filter

//it will change the velocity depends on the input audio signal

Transfer function of filter bandwidth of developed TFVBF is calculated

$$
\begin{aligned}
V_{a-1}^{a}=\frac{S_{a}}{S_{a-1}}= & \frac{1}{Q_{a} P_{a}}\left(P_{a-1}\right. \\
& \left.+\sum_{b=a-k_{d}}^{a-2} \frac{X_{a, b}}{H_{a, b}} \frac{1}{\prod_{i=k}^{a-2} B_{i}^{i+1}}\right)
\end{aligned}
$$

Replacement of multiplier

// for reducing complexity, replace multipliers using adder and shifter

\section{Estimated filter parameter}

\section{Optimize HAS}

// reducing error, delay, complexity, and matching error

\}

End 


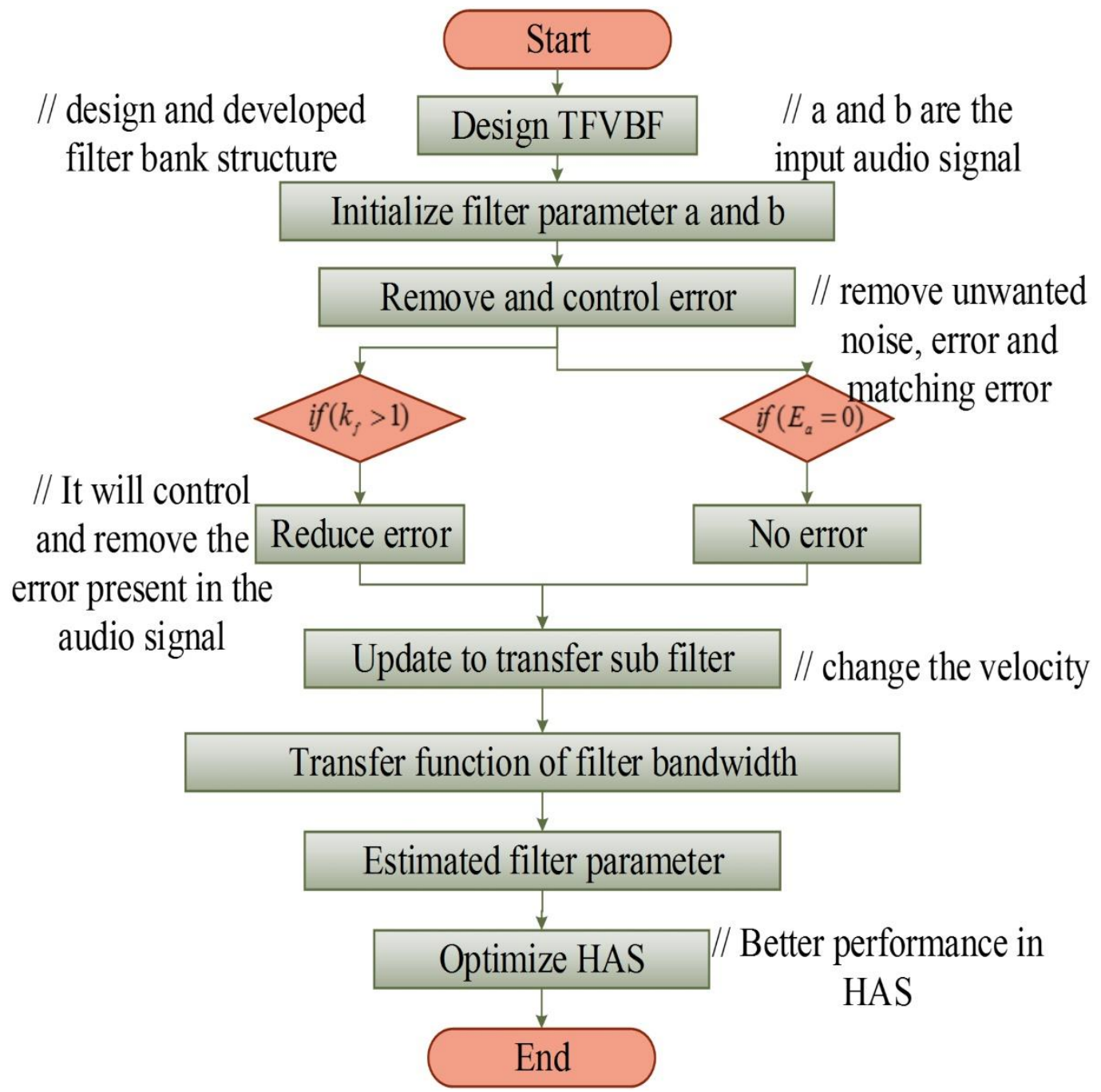

Figure. 5 Workflow of proposed TFVBF technique

\section{Results and discussion}

Generally, a novel variable bandwidth filter is designed and developed for optimizing the performance of HAS. Moreover, the developed TFVBF technique is implemented in MATLAB platform. The performance metrics of the developed technique is compared with other existing replicas in terms of frequency response, matching error, delay, power consumption, and complexity.

\subsection{Case study}

Generally, hearing loss affects human life, work, relationships, emotionally, and physically it is a significant health problem of human life. So in this research, the TFVBF technique is developed to optimize hearing aid to enhance hearing ability and remove unwanted noise and reduce complexity. Thus the proposed filter bank is used to remove the errors, noise and reduce complexity. Moreover, the hearing losses are denoted as moderate, severe, normal, and mild. Initially, trained input audio signals are given to the developed replica, and the input audio signal is converted into a digital signal with the help of a designed filter. Thus the digital signal transfers into the Digital Signal Processing (DSP). This section will reduce the unwanted noise, delay, error, matching error, and power dissipation presented in the audio signal. Furthermore, it will minimize the complexity based on the replacement of the multiplier. The developed TFVBF process has illustrated in Fig. 6.

Let us consider 20 bands of filters are designed for enhancing the performance of TFVBF from unwanted noise, delay, error, complexity, and matching error. In the filtering process, it will remove the errors, noise, and less complexity. Moreover, developed replica gain more efficiency in HAS and less power consumption. Additionally, developed TFVBF replica is operated various bands for separating audiogram matching. Thus the developed TFVBF attain a low rate in power consumption, error, unwanted noise, matching error, 


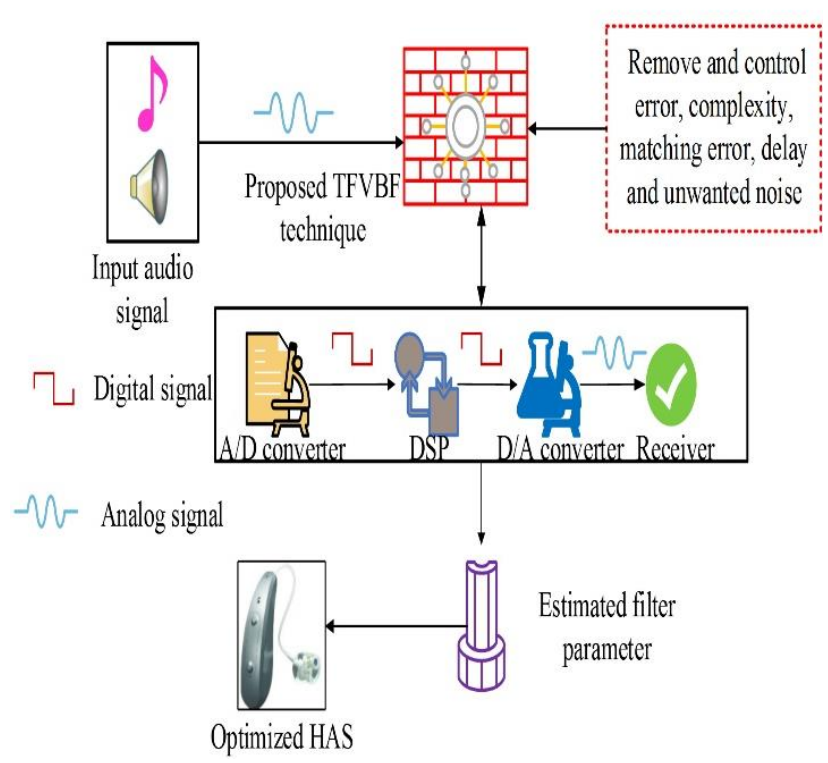

Figure. 6 Process of TFVBF technique

Table 1. Specification of designed filter

\begin{tabular}{|l|l|l|l|l|l|}
\hline $\begin{array}{l}\text { Numb } \\
\text { er of } \\
\text { slide } \\
\text { lobes }\end{array}$ & $\begin{array}{l}\text { Width of } \\
\text { transition } \\
\text { band } \\
\text { (rad } / \boldsymbol{\pi})\end{array}$ & $\begin{array}{l}\text { Maximu } \\
\text { m pass } \\
\text { band } \\
\text { ripple } \\
\text { (dB) }\end{array}$ & $\begin{array}{l}\text { Maximu } \\
\text { m stop } \\
\text { band } \\
\text { attenuat } \\
\text { ion }(\mathbf{d B})\end{array}$ & $\begin{array}{l}\text { Total } \\
\text { out of } \\
\text { band } \\
\text { power } \\
\text { (dB) }\end{array}$ & $\begin{array}{l}\text { Num } \\
\text { ber } \\
\text { of } \\
\text { adde } \\
\text { r }\end{array}$ \\
\hline 4 & 955.25 & 0.0806 & -48.05 & 0.555 & 347 \\
\hline 8 & 1052 & 0.0806 & -48.03 & 0.87 & 352 \\
\hline 10 & 1090 & 0.0802 & -47.89 & 0.456 & 333 \\
\hline 13 & 1156.3 & 0.0774 & -47.76 & 0.432 & 325 \\
\hline 15 & 1190.03 & 0.0729 & -36.70 & 0.399 & 300 \\
\hline 18 & 1250.23 & 0.0719 & -62.54 & 0.365 & 402 \\
\hline 20 & 1312.4 & 0.0699 & -61.01 & 0.345 & 432 \\
\hline 22 & 1390 & 0.0687 & -555.67 & 0.330 & 456 \\
\hline
\end{tabular}

and complexity. As a result, the designed TFVBF technique achieved better performance in HAS.

The designed filter used slide is 4 to 22 , and the adder range is 347 . Also, the width of the transition band is 955.25 , and the maximum pass band and stop band ripple are 0.0806 and -48.05 . Additionally, the total band power is 0.555 , and the specification of the designed filter is detailed in the Table 1.

\subsection{Performance metrics}

The performance of the proposed TFVBF has been examined with obtained parameter validation, and the achieved performance metrics are compared with other existing techniques in terms of frequency response, matching error, complexity, delay, and power consumption. Moreover, the developed technique is implemented in MATLAB, and the performance metrics are compared with some existing replicas that are Narrow Transition Band (NTB) [26], Octave Interpolated Filter (OIP) [21], Analog Correlator (AC) [25], Synthesis of Filter
Bank (SFB) [22], Restoration Audibility in HA (RAHA) [23], Least Square Dynamic Filter (LSDF) [27], Continuous VBF (CVBF) [28] and Interpolated Finite Impulse response (IFIR) [24].

\subsubsection{Complexity}

The number of required resources for running MATLAB will also change the input size, and the complexity is denoted as $f(n)$. Where $n$ is the input size and $f(n)$ are represented as the worst case of complexity which is the maximum amount of the needed resource of every input size. Thus the complexity is calculated by the multiple numbers of times for executed program repeated in a loop. Moreover, the comparison of complexity is detailed in Table 2.

Generally, the achieved complexity of developed TFVBF is compared with other existing replicas such as NTB, OIP, LSDF, and RAHA. The existing technique of RAHA and NTB achieved $30 \%$ for using multiplier as 0.2 , and OIP replica achieved $40 \%$ complexity. Furthermore, the LSDF technique earned $50 \%$, but the developed TFVBF replica gained a low level in complexity.

Thus the proposed TFVBF technique has achieved a complexity rated is $25 \%$ it is low while comparing another replica. Additionally, the

Table 2. Comparison of complexity

\begin{tabular}{|c|c|c|c|c|c|}
\hline $\begin{array}{c}\text { Amount of } \\
\text { multipliers }\end{array}$ & \multicolumn{5}{|c|}{ Complexity (\%) } \\
\cline { 2 - 6 } & NTB & OIP & RAH & LSDF & $\begin{array}{c}\text { TFVBF } \\
\text { [proposed] }\end{array}$ \\
\hline 0.2 & 30 & 40 & 30 & 50 & 25 \\
\hline 0.4 & 57 & 59 & 63 & 65 & 36 \\
\hline 0.6 & 36 & 75 & 138 & 45 & 30 \\
\hline 0.8 & 52 & 80 & 84 & 68 & 45 \\
\hline 0.10 & 61.11 & 92 & 67 & 74 & 50 \\
\hline
\end{tabular}

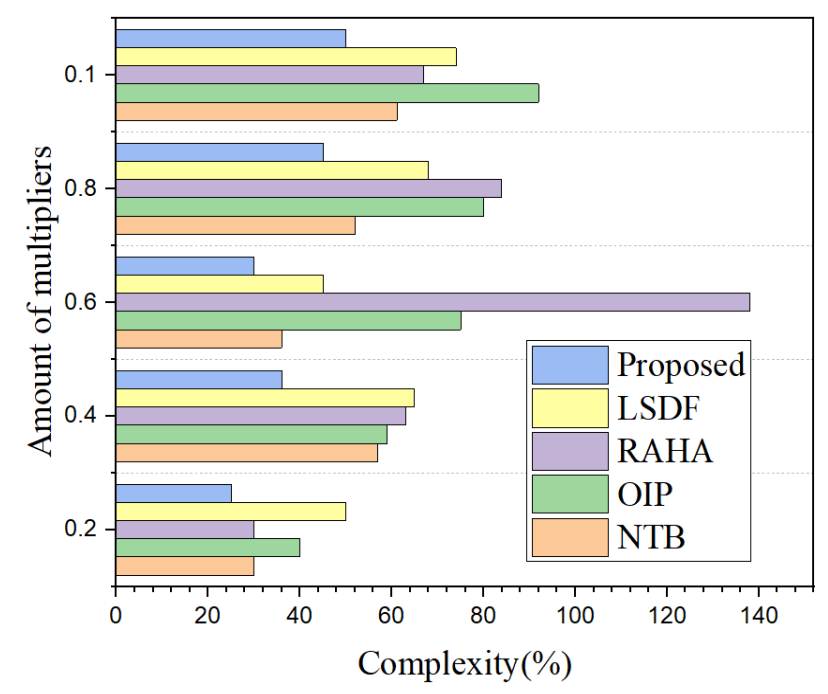

Figure. 7 Complexity comparison 
complexity comparison with existing technique has elaborated in Fig. 7.

\subsubsection{Delay}

The calculation of delay is the most wanted parameter in the filter bank of HAS. Thus the performance of the delay values is identified based on low and high values. Moreover, the delay is the ratio of several multipliers in a single band through the number of sub-filters in the band, and the measurement of delay is obtained using Eq. (11).

$$
\text { Delay }=\frac{A}{S}
$$

$A$ is denoted as amount of single multiplier and $S$ is represented as amount of sub filters in the band. Moreover, the comparison of delay rate is detailed in Table 3.

Table 3. Comparison of delay

\begin{tabular}{|c|c|c|c|c|c|}
\hline \multirow{2}{*}{$\begin{array}{c}\text { Amount } \\
\text { of bands }\end{array}$} & \multicolumn{5}{|c|}{ Delay (ms) } \\
\cline { 2 - 6 } & SFB & OIP & CVBF & IFIR & $\begin{array}{c}\text { TFVBF } \\
\text { [Proposed] }\end{array}$ \\
\hline 10 & 18.61 & 31 & 29 & 48 & 15 \\
\hline 20 & 13.56 & 10 & 25 & 10 & 5 \\
\hline 30 & 34.7 & 26.6 & 36 & 13.58 & 10 \\
\hline 40 & 56 & 18.5 & 45 & 10 & 8 \\
\hline 50 & 43 & 21.6 & 39 & 9.75 & 7 \\
\hline
\end{tabular}

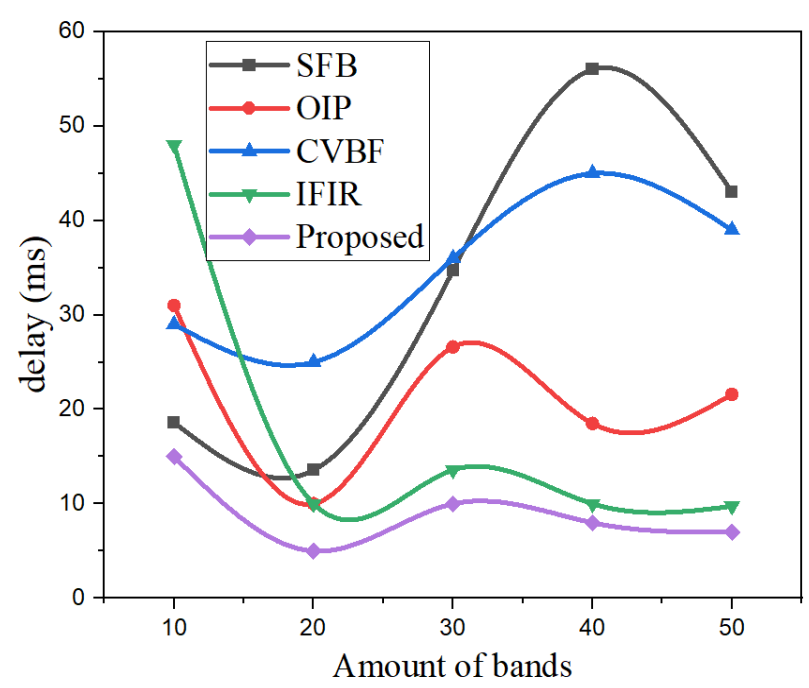

Figure. 8 delay comparison

In better performance, the delay rate is low. Initially, the uniform filter bank contains less delay while comparing non-uniform filter banks. Subsequently, the high delay affects the capability of lip reading of hearing damage patients, and the delay rate comparison with the existing technique has elaborated in Fig. 8.
The proposed TFVBF replica attained a delay rate is $7 \%$ of 50 bands, and the existing replica of SFB and OIP achieved $43 \mathrm{~ms}$ and $21.6 \mathrm{~ms}$ in delay. Furthermore, the IFIR replica earned $9.75 \mathrm{~ms}$ in delay rate, and the CVBF attained $39 \mathrm{~ms}$ in delay for 50 bands. Compare to another existing replica, the proposed TFVBF technique attained a low rate of delay.

\subsubsection{Matching error}

Initially, matching error is the difference among measurement of audiograms and matching curves of hearing damage patients. Thus the computation of the matching error is useful for identifying the capability of developed filter bank structure, and the comparison of matching error has illustrated in Fig. 9.

Generally, the achieved matching error of developed TFVBF is compared with another existing replica such as SFB, RAHA, LSDF, and CVBF. The existing technique of RAHA achieved $4.23 \mathrm{~dB}$ in 10 bands, and the SFB replica gained $2.09 \mathrm{~dB}$ of matching error for using ten bands, and the LSDF replica achieved a $1.24 \mathrm{~dB}$ rate in matching error. Furthermore, the CVBF technique earned $1.40 \mathrm{~dB}$ for ten bands, but the developed TFVBF replica gained a low matching error rate.

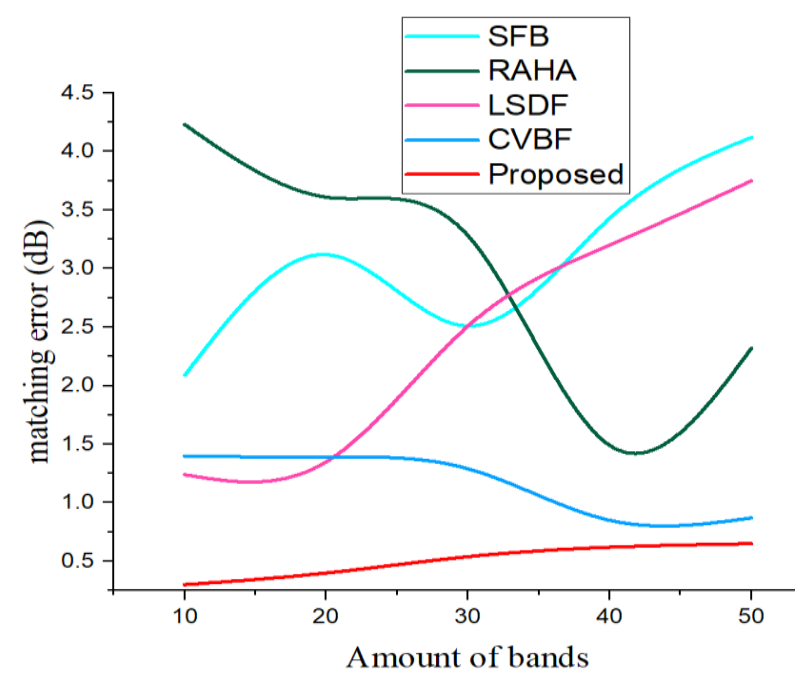

Figure. 9 Matching error comparison

Table 4. Comparison of matching error

\begin{tabular}{|c|c|c|c|c|c|}
\hline \multirow{2}{*}{$\begin{array}{c}\text { Amount } \\
\text { of bands }\end{array}$} & \multicolumn{5}{|c|}{ Matching error (dB) } \\
\cline { 2 - 6 } & SFB & RAHA & LSDF & CVBF & $\begin{array}{c}\text { TFVBF } \\
\text { [Proposed] }\end{array}$ \\
\hline 10 & 2.09 & 4.23 & 1.24 & 1.40 & 0.3 \\
\hline 20 & 3.12 & 3.61 & 1.35 & 1.39 & 0.4 \\
\hline 30 & 2.51 & 3.28 & 2.51 & 1.29 & 0.54 \\
\hline 40 & 3.43 & 1.49 & 3.2 & 0.85 & 0.62 \\
\hline 50 & 4.12 & 2.32 & 3.75 & 0.87 & 0.65 \\
\hline
\end{tabular}


Additionally, the matching error comparison had described in Table 4.

Thus the proposed TFVBF technique achieved a matching error rate is as $0.3 \mathrm{~dB}$ for using ten bands. While comparing other replicas, the developed TFVBF technique gained a low rate of matching error. As a result, enhance the performance of hearing aid.

\subsubsection{Power consumption}

Power consumption $(p)$ is the ratio of energy and time consumed to filter the issues such as unwanted noise, errors, and matching errors. The consumption of power can be done in voltage. The mathematical representation of power consumption is measured using Eq. (12).

$$
P=\frac{E}{t}
$$

$E$ is represented as the energy consumption of the developed TFVBF technique for filtering the signal, and $t$ is expressed as the time taken for filtering the input audio signal. Moreover, the comparison of power consumption has been illustrated in Fig. 10.

The proposed TFVBF replica attained a delay rate is $7 \%$ of 50 bands, and the existing replica of SFB and OIP achieved $43 \mathrm{~ms}$ and $21.6 \mathrm{~ms}$ in delay. Furthermore, the IFIR replica earned $9.75 \mathrm{~ms}$ in delay rate, and the CVBF attained $39 \mathrm{~ms}$ in wait for 50 bands. Compared to other existing models, proposed TFVBF technique achieved a low rate of delay. Moreover, the comparison of power consumption with existing replica has been illustrated in Table 5.

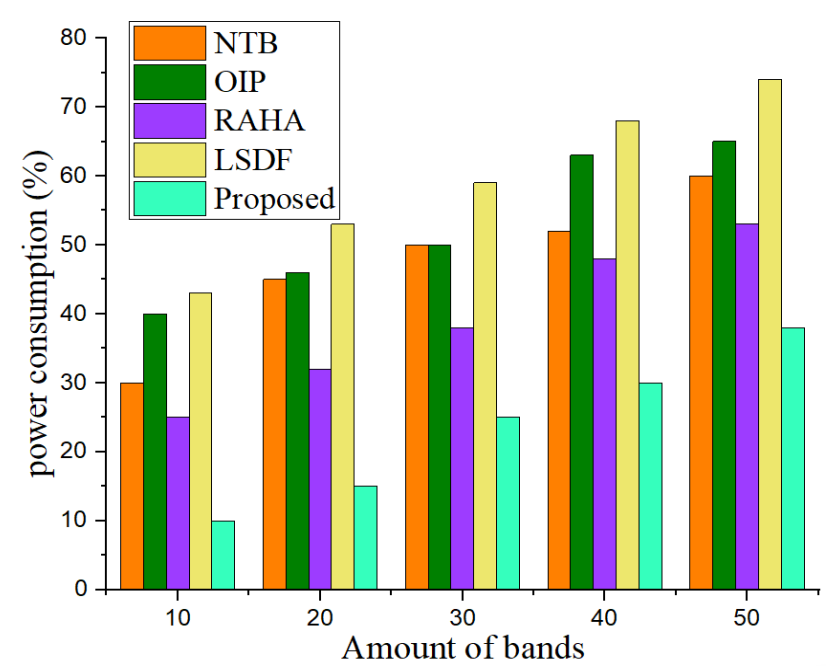

Figure. 10 Power consumption comparison
Table 5. Comparison of power consumption

\begin{tabular}{|c|c|c|c|c|c|}
\hline \multirow{2}{*}{$\begin{array}{c}\text { Amount } \\
\text { of bands }\end{array}$} & \multicolumn{5}{|c|}{ Power consumption (\%) } \\
\cline { 2 - 6 } & NTB & OIP & RAHA & LSDF & $\begin{array}{c}\text { TFVBF } \\
\text { [Proposed] }\end{array}$ \\
\hline 10 & 30 & 40 & 25 & 43 & 10 \\
\hline 20 & 45 & 46 & 32 & 53 & 15 \\
\hline 30 & 50 & 50 & 38 & 59 & 25 \\
\hline 40 & 52 & 63 & 48 & 68 & 30 \\
\hline 50 & 60 & 65 & 53 & 74 & 38 \\
\hline
\end{tabular}

The performance metrics of the developed TFVBF technique achieve better performance for optimizing the hearing aid system, and it will reduce the error, unwanted noise, matching error, and complexity. Thus the developed TFVBF method is most helpful for hearing loss patients.

\subsection{Discussion}

In the overall assessment, the proposed TFVBF has achieved good performance in matching error and power consumption. The complexity and delay rate are less compared to other existing techniques. Also, it will remove the unwanted noise and generate an accurate audio signal. Thus the developed replica enhances the performance of the hearing aid system. It will help hearing loss patients for communication.

\section{Conclusions}

The most challenging task of hearing loss is reducing risk in the hearing aid system, and hearing aid issues are error, delay, complexity, power dissipation, and high frequency. This paper developed and designed a novel TFVBF technique for enhancing hearing aid performance and reducing risk obtained in hearing aid. Thus the designed filter parameter is reducing the delay, matching error, power dissipation, and complexity. In this technique, several sub filters are used to optimize HAS, and the replacement of multiplier reduces the complexity. The main aim of the developed technique is to minimize the risk, and proper communication to the hearing impair patients. Moreover, validate the performance metrics of the developed technique with other methods to identify the proposed framework's efficiency. Additionally, the developed TFVBF method attained a low matching error of 0.3 $\mathrm{dB}$ for ten bands, and power consumption gained $10 \%$. As well proposed technique achieved $25 \%$ of complexity it is low compared to another existing replica. In the future, a hybrid variable bandwidth filter have developed for attaining better outcomes such as matching error, power consumption, noise 
reduction in HAS. Also, it will enhance the performance of HA.

\section{Conflicts of Interest}

The authors declare no conflict of interest.

\section{Author Contributions}

Ujjwala Rawandale, the first author contributed in conceptualization, methodology, software, validation, formal analysis, investigations, writing original draft preparation, writing - review and editing and visualization. Mahesh Kolte, the second authors supervised and administered project.

\section{References}

[1] N. Rana, M. S. A. Latiff, and H. Chiroma, "Whale optimization algorithm: a systematic review of contemporary applications, modifications and developments", Neural Computing and Applications, pp. 1-33, 2020.

[2] W. G. Hatcher and W. Yu, "A survey of deep learning: Platforms, applications and emerging research trends", IEEE Access, Vol. 6, pp. 24411-24432, 2018.

[3] W. Huang, H. Sun, J. Luo, and W. Wang, "Periodic feature oriented adapted dictionary free OMP for rolling element bearing incipient fault diagnosis", Mechanical Systems and Signal Processing, Vol. 126, pp. 137-160, 2019.

[4] S. Krishnan and Y. Athavale, "Trends in biomedical signal feature extraction", Biomedical Signal Processing and Control, Vol. 43, pp. 41-63, 2018.

[5] H. Miyamoto, S. Shiota, and H. Kiya, "Nonlinear harmonic generation based blind bandwidth extension considering aliasing artifacts", In: Proc. of 2018 Asia-Pacific Signal and Information Processing Association Annual Summit and Conference, 2018.

[6] H. Fu, S. Abeywickrama, L. Zhang, and C. Yuen, "Low-complexity portable passive drone surveillance via SDR-based signal processing", IEEE Communications Magazine, Vol. 56, No. 4, pp. 112-118, 2018.

[7] K. Kim, "Preliminary Digital Filter Design", Conceptual Digital Signal Processing with MATLAB, Springer, Singapore, pp. 1-19, 2021.

[8] C. K. A. Reddy, N. Shankar, G. S. Bhat, R. Charan, and I. Panahi, "An individualized super-Gaussian single microphone speech enhancement for hearing aid users with smartphone as an assistive device", IEEE
Signal Processing Letters, Vol. 24, No. 11, pp. 1601-1605, 2017.

[9] L. Pisha, J. Warchall, T. Zubatiy, S. Hamilton, C. H. Lee, G. Chockalingam, P. P. Mercier, R. Gupta, B. D. Rao, and H. Garudadri, "A wearable, extensible, open-source platform for hearing healthcare research", IEEE Access, Vol. 7, pp. 162083-162101, 2019.

[10] H. A. Hejazi, and H. M. Rad, "Power systems big data analytics: An assessment of paradigm shift barriers and prospects", Energy Reports, Vol. 4, pp. 91-100, 2018.

[11] P. A. Harris, R. Taylor, B. L. Minor, V. Elliott, M. Fernandez, L. O'Neal, L. McLeod, G. Delacqua, F. Delacqua, J. Kirby, and S. N. Duda, "The REDCap consortium: Building an international community of software platform partners", Journal of Biomedical Informatics, Vol. 95, pp. 103208, 2019.

[12] G. S. Bhat, N. Shankar, and I. M. S. Panahi, "Automated machine learning based speech classification for hearing aid applications and its real-time implementation on smartphone", In: Proc. of 2020 42nd Annual International Conference of the IEEE Engineering in Medicine \& Biology Society, 2020.

[13] M. Chen, Y. Miao, Y. Hao, and K. Hwang, "Narrow band internet of things", IEEE Access, Vol. 5, pp. 20557-20577, 2017.

[14] X. Wang, X. Zha, W. Ni, R. P. Liu, Y. J. Guo, $X$. Niu, and K. Zheng, "Survey on blockchain for Internet of Things", Computer Communications, Vol. 136, pp. 10-29, 2019.

[15] R. D. P. Reddy and V. Sharma, "Additive manufacturing in drug delivery applications: A review", International Journal of Pharmaceutics, Vol. 589, pp. 119820, 2020.

[16] L. Lu, X. Zhang, and X. Gao, "Nonimplantable Artificial Hearing Technology", Hearing Loss: Mechanisms, Prevention and Cure, pp. 145-163, 2019.

[17] B. Mishachandar and S. Vairamuthu, "An underwater cognitive acoustic network strategy for efficient spectrum utilization", Applied Acoustics, Vol. 175, pp. 107861, 2021.

[18] R. A. Kassas, M. Bansal, and J. Shaw, "Nanosizing techniques for improving bioavailability of drugs", Journal of Controlled Release, Vol. 260, pp. 202-212, 2017.

[19] H. Yang, G. P. Jiang, W. K. S. Tang, G. Chen, and Y. C. Lai, "Multi-carrier differential chaos shift keying system with subcarriers allocation for noise reduction", IEEE Transactions on Circuits and Systems II: Express Briefs, Vol. 65, No. 11, pp. 1733-1737, 2017. 
[20] H. Wang, Y. Jin, and J. Doherty, "Committeebased active learning for surrogate-assisted particle swarm optimization of expensive problems", IEEE Transactions on Cybernetics, Vol. 47, No. 9, pp. 2664-2677, 2017.

[21] T. Devis and M. Manuel, "Hardware-efficient auto-reconfigurable hearing aids using 3-level octave interpolated filters for auditory compensation applications", Physical and Engineering Sciences in Medicine, pp. 1-14, 2021.

[22] V. V. Mahesh and T. K. Shahana, "Design and synthesis of filter bank structures based on low order constrained least square and minimum phase methods for audiogram matching in digital hearing aids", Health and Technology, Vol. 11, No. 1, pp. 153-168, 2021.

[23] T. Devis and M. Manuel, "A low-complexity 3level filter bank design for effective restoration of audibility in digital hearing aids", Biomedical Engineering Letters, Vol. 10, No. 4, pp. 593-601, 2020.

[24] S. P. Deepu and R. Kini, "18 band ANSI S1. 11 filter bank based on interpolated finite impulse response technique for hearing aids", The Journal of Engineering, Vol. 2020, No. 9, pp. 760-767, 2020.

[25] H. Park, S. Jung, and H. J. Chung, "An analog correlator based CMOS analog front end with digital gain control circuit for hearing aid devices", Analog Integrated Circuits and Signal Processing, Vol. 105, No. 2, pp. 157$165,2020$.

[26] S. Roy and A. Chandra, "Design of narrow transition band variable bandwidth digital filter", IET Circuits, Devices \& Systems, Vol. 14, No. 6, pp. 750-757, 2020.

[27] V. V. Mahesh and T. K. Shahana, "Constrained least square nonuniform dynamic filter bank for delay and Hardware efficient digital hearing aids", Health and Technology, Vol. 9, No. 3, pp. 355-363, 2019.

[28] R. Indrakanti, N. Haridas, and E. Elias, "High performance continuous variable bandwidth digital filter design for hearing aid application", AEU-International Journal of Electronics and Communications, Vol. 92, pp. 36-53, 2018. 